\title{
Informations
}

Orthop Traumatol (1992) $2: 214$

\section{Janvier 1993}

\section{Séminaire National du Collège Français des Chirurgiens Orthopédistes Traumatologues}

Strasbourg, 8 et 9 janvier 1993

Sujets : $\quad$ Chirurgie du rachis cervical traumatique et dégénératif
sous la direction du Pr. P. Kehr et du Dr. A. Grosse
- La prothèse totale de hanche sans ciments
sous la direction du Pr. I. Kempf et du Dr. D. Dagrenat

Cours ouvert aux auditeurs à l'enseignement du Collège, aux membres du Collège Français des Chirgriens Orthopédiques et Traumatologues ainsi qu'aux membres de le S.O.T.EST et du GECO

Renseignements, inscriptions : AIOD-FORMATION, 3, rue du Général Offenstein, F-67100 Strasbourg, Té1. 883962 90, Fax 88394396

\section{Juin 1993}

XXXVIIe Réunion de la Société d'OrthopédieTraumatologie de l'Est de la France (S.O.T.EST) Congrès Européen

\section{Strasbourg, 11 au 13 juin 1993}

\section{Sujets des Tables Rondes :}

-Agents antimicrobiens dans le traitement local de l'infection ostéo-articulaire

- Que reste-t'il du traitement non chirurgical de la scoliose idiopathique en période de croissance?

Renseignements et inscriptions : AIOD-FORMATION, 3, rue du Général Offenstein, F-67100 Strasbourg, Tél. 883962 90, Fax 88394396
XVIIe Réunion Elargie du Groupe d'Etude de Chirurgie Osseuse (GECO)

Les Ares (Bourg-Saint-Maurice), 16 au 23 janvier 1993

Sujets des Tables Rondes:

- Epiphyse et apophyse de l'enfant et traumatismes

- Rotule douloureuse non instable

Renseignements et inscriptions : GECO, 9, rue Guillaume Tell, BP 1232, F68054 Mulhouse Cedex, Tél. 89360532 